\title{
GERAÇÃO E CONTROLE DAS CORES LUZ PRIMÁRIAS EM VIDROS PARA DISPOSITIVOS "FULL COLOR"
}

José Expedito C. Silva, Oscar L. Malta, Gilberto F. de Sá e Petrus A. Santa-Cruz

Departamento de Química Fundamental - LVC - CCEN - Universidade Federal de Pernambuco - Cid. Universitária - 50670-901

- Recife - PE

Recebido em 2/12/96; aceito em 19/5/97

\begin{abstract}
GENERATION AND CONTROL OF THE PRIMARY LIGHT COLORS IN GLASSES FOR FULL COLOR DEVICES. We are working in a new multi-doped glassy material to generate simultaneously the three primary light color (by addition) of the visible spectrum, with the control of the intensity of each one, allowing the simulation of any color: a full-color generator device. $\mathrm{Tm}^{+3}$, $\mathrm{Tb}^{+3}$ and $\mathrm{Eu}^{+3}$ ions were used $(0.01$ to $5,0 \mathrm{~mol} \%)$ as blue, green and red narrow line emitters. A wide color gamut was obtained under ultraviolet excitation by varying the material composition. The chromaticity diagram is covered, including the white simulation.
\end{abstract}

Keywords: tunable light synthesis; multi-doped glass; chromaticy control.

\section{INTRODUÇÃO}

A geração e controle de intensidade relativa das cores luz primárias (aditivas), no estado sólido, tem se mostrado de grande importância para o desenvolvimento de dispositivos que permitam alta resolução de imagens, usados em monitores coloridos, displays planos ou tridimensionais, dispositivos optoeletrônicos, entre outros. Várias publicações buscam a geração dessas cores, bem como o controle independente da cromaticidade $^{1,2}$. Neste trabalho desenvolvemos um novo material vítreo $\left(\mathrm{CaF}_{2}-\mathrm{B}_{2} \mathrm{O}_{3}-\mathrm{Sb}_{2} \mathrm{O}_{3}\right)$ multidopado com íons de terras-raras $\left(\mathrm{Tm}^{+3}, \mathrm{~Tb}^{+3}\right.$ e Eu$\left.{ }^{+3}\right)$, capaz de gerar simultaneamente as cores azul, verde e vermelho. Propomos ainda métodos para o controle da cromaticidade através do uso do íon $\mathrm{Er}^{+3}$ incorporado à matriz, atuando como supressor de luminescência (quencher), no vidro com concentração fixa da tríade de íons de terras-raras ${ }^{3.4}$.

O vidro desenvolvido, quando excitado por radiação ultravioleta, produz a sensação de uma cor-luz predefinida qualquer do diagrama de cromaticidade (CIE), inclusive a simulação de luz branca pela composição das cores primárias.

\section{EXPERIMENTAL}

As amostras usadas neste trabalho foram preparadas mediante mistura de $\mathrm{B}_{2} \mathrm{O}_{3}\left(99,999 \%\right.$ Aldrich), $\mathrm{CaF}_{2}$ (99,99\% Aldrich), $\mathrm{Sb}_{2} \mathrm{O}_{3}$ (99,99\% Merck), $\mathrm{Eu}_{2} \mathrm{O}_{3}$ (99,999\% Aldrich), $\mathrm{TbCl}_{3}$ (partindo do $\mathrm{Tb}_{4} \mathrm{O}_{7} 99,999 \%$ Aldrich), $\mathrm{Tm}_{2} \mathrm{O}_{3}$ (99,999\% Aldrich), levados à fusão em cadinho de platina a $1200^{\circ} \mathrm{C}$ com posterior tratamento térmico a $400^{\circ} \mathrm{C}$ durante uma hora em molde de carbono vítreo ${ }^{5}$.

A composição da amostra, em mol\%, para a formação do vidro é dada por:

$72.0 \mathrm{~B}_{2} \mathrm{O}_{3}-27.0 \mathrm{CaF}_{2}-1.0 \mathrm{Sb}_{2} \mathrm{O}_{3}: \mathrm{x} \mathrm{Tm}_{2} \mathrm{O}_{3}-\mathrm{y} \mathrm{Eu}_{2} \mathrm{O}_{3}-\mathrm{z}$ $\mathrm{Tb}_{2} \mathrm{O}_{3}$, x, y e $\mathrm{z}$ variando de 0,01 a $5,0 \mathrm{~mol} \%$.

A análise espectral (espectros de emissão e excitação) foi obtida a temperatura ambiente através de um espectrômetro Jobin-Yvon modelo Ramanor U-1000 com duplo monocromador, usando como fonte de excitação lâmpadas de Xe $(400 \mathrm{~W})$ ou Xe-Hg $(150 \mathrm{~W})$, com detecção através de fotomultiplicadora RCA C31034-RF. As amostras foram analisadas em blocos $(1.0 \times 1.0 \times 0.2 \mathrm{~cm})$ e em pó, para monitoração de eventuais processos de reabsorção de energia.

\section{RESULTADOS E DISCUSSÃO}

A produção de cores-luz secundárias no vidro é obtida pela dupla dopagem: $\mathrm{Eu}^{+3}\left(0,30 \mathrm{~mol} \%\right.$ do óxido) e $\mathrm{Tb}^{+3}(2,70 \mathrm{~mol} \%$ do cloreto) para amarelo, $\mathrm{Tb}^{+3}\left(0,14 \mathrm{~mol} \%\right.$ do cloreto) e $\mathrm{Tm}^{+3}$ $\left(0,20 \mathrm{~mol} \%\right.$ do óxido) para ciano e $\mathrm{Tm}^{+3}(0,20 \mathrm{~mol} \%$ do óxido) e $\mathrm{Eu}^{+3}(0,20 \mathrm{~mol} \%$ do óxido) para magenta. Pela variação das concentrações dos íons $\mathrm{Tm}^{+3}, \mathrm{~Tb}^{+3}$ e $\mathrm{Eu}^{+3}$ no vidro multidopado, é possível simular qualquer cor do diagrama de cromaticidade (CIE). O espectro de luminescência típico desses vidros mostra cinco linhas de emissão no visível correspondente às transições: ${ }^{1} \mathrm{D}_{2} \rightarrow{ }^{3} \mathrm{~F}_{4}(453 \mathrm{~nm})$ do íon $\mathrm{Tm}^{+3}$, ${ }^{5} \mathrm{D}_{4} \rightarrow{ }^{7} \mathrm{~F}_{5}(542 \mathrm{~nm})$ e ${ }^{5} \mathrm{D}_{4} \rightarrow{ }^{7} \mathrm{~F}_{6}(488 \mathrm{~nm})$ do íon $\mathrm{Tb}^{+3}$ superposição das transições ${ }^{5} \mathrm{D}_{0} \rightarrow{ }^{7} \mathrm{~F}_{2}$ do íon $\mathrm{Eu}^{+3}$ e ${ }^{5} \mathrm{D}_{4} \rightarrow{ }^{7} \mathrm{~F}_{3}$ do íon $\mathrm{Tb}^{+3}(615 \mathrm{~nm}),{ }^{5} \mathrm{D}_{0} \rightarrow{ }^{7} \mathrm{~F}_{0,1}$ do íon $\mathrm{Eu}^{+3}$ e ${ }^{5} \mathrm{D}_{4} \rightarrow{ }^{7} \mathrm{~F}_{4}$ do íon $\mathrm{Tb}^{+3}(586 \mathrm{~nm})$. A figura 1 mostra a emissão do vidro projetado para simular luz branca quando excitado em $365 \mathrm{~nm}$.

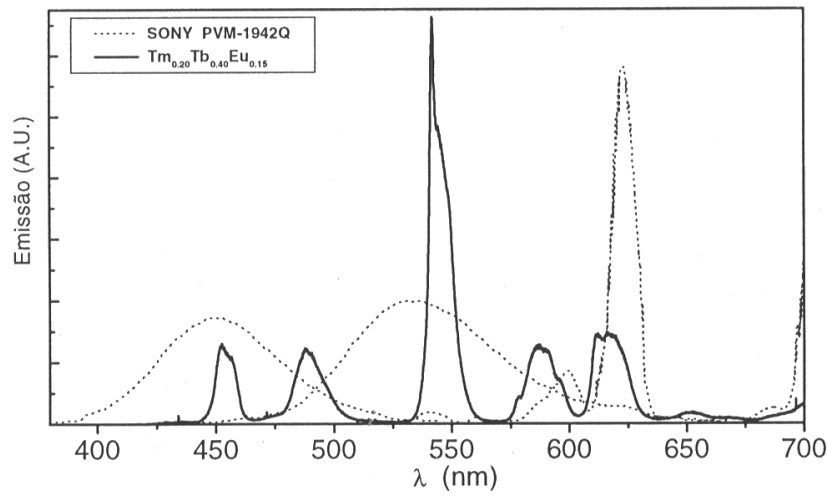

Figura 1. Comparação entre a emissão de um monitor padrão SONY (linha pontilhada) e a luz branca emitida pelo vidro multidopado (linha sólida) quando excitado na região do $U V(365 \mathrm{~nm})$.

Devido aos diversos mecanismos de transferência de energia e às diferentes probabilidades de transições envolvendo os íons de terras-raras, a concentração dos óxidos de $\mathrm{Tm}^{+3}$ e $\mathrm{Eu}^{+3}$ e cloreto de $\mathrm{Tb}^{+3}$ para a geração de luz branca no vidro é de $0,20,0,15$ e $0,40 \mathrm{~mol} \%$ respectivamente. $\mathrm{O}$ íon $\mathrm{Tm}^{+3}$, usado na geração da cor-luz azul, produz uma emissão de largura espectral relativamente fina quando comparada com as bandas 
largas emitidas por fósforos usados comercialmente em tubos de televisão colorida, o íon $\mathrm{Tb}^{+3}$ produz também uma emissão de largura espectral fina centrada na região do verde (fig. 1).

A concentração máxima do íon $\mathrm{Tm}^{+3}$ na amostra com composição fixa, para simulação de luz branca, foi monitorada por medidas de tempo de vida evitando os efeitos de auto supressão de luminescência (self quenching) causados por processos de relaxação cruzada. Em consequiência, as concentrações de $\mathrm{Eu}^{+3} \mathrm{e} \mathrm{Tb}^{+3}$ foram calculadas em função da concentração fixa do íon $\mathrm{Tm}^{+3}$.

A figura 2 corresponde ao espectro de excitação, com observação centrada em 453, 542 e $615 \mathrm{~nm}$, respectivamente, para a amostra com composição projetada para gerar luz branca. Esses espectros mostram a intensidade das cores primárias em função do comprimento de onda da radiação incidente. Os espectros em linhas sólidas mostram o efeito do $\mathrm{Er}^{+3}$ como supressor da luminescência dos três íons emissores. A eficiência da supressão é função do comprimento de onda de excitação. Em torno de 370 $n m$ a supressão da emissão do íon $\mathrm{Tb}^{+3}$ é visivelmente mais eficiente quando comparado aos íons $\mathrm{Eu}^{+3} \mathrm{e} \mathrm{Tm}^{+3}$. Esta seletividade pode ser usada para obtenção de um sistema sintonizável. Uma segunda fonte de excitação pode ser usada, preferencialmente na região do infravermelho, de forma a excitar o íon $\mathrm{Er}^{+3}$ e reduzir sua função de supressor. Resultados detalhados deste processo serão publicados posteriormente, incluindo aplicações deste novo material em dispositivos luminescente de alta resolução e sensores seletivos. Outros processos de excitação, incluindo eletroluminescência, também estão sendo estudados.

\section{CONCLUSÃO}

Desenvolvemos neste trabalho um novo material vítreo, multidopado unicamente com íons de terras-raras, capaz de gerar as três cores-luz primárias com larguras espectrais particularmente finas. Este material possibilita a simulação de qualquer cor do diagrama de cromaticidade (CIE), em função da concentração relativa dos íons de terras-raras $\left(\mathrm{Tm}^{+3}, \mathrm{~Tb}^{+3} \mathrm{e} \mathrm{Eu}^{+3}\right)$. Numa primeira etapa para o controle da cromaticidade em uma composição fixa do material, usamos o íon $\mathrm{Er}^{+3}$ como supressor de luminescência. A eficiente seletividade de supressão de luminescência do íon $\mathrm{Tb}^{+3}$ é detectada, particularmente em torno de $370 \mathrm{~nm}$, o que mostra ser o sistema muito promissor para mecanismos de controle de cromaticidade envolvendo excitação múltipla.

\section{AGRADECIMENTOS}

Os autores agradecem ao CNPq, CAPES e ao FINEP pelo suporte financeiro.

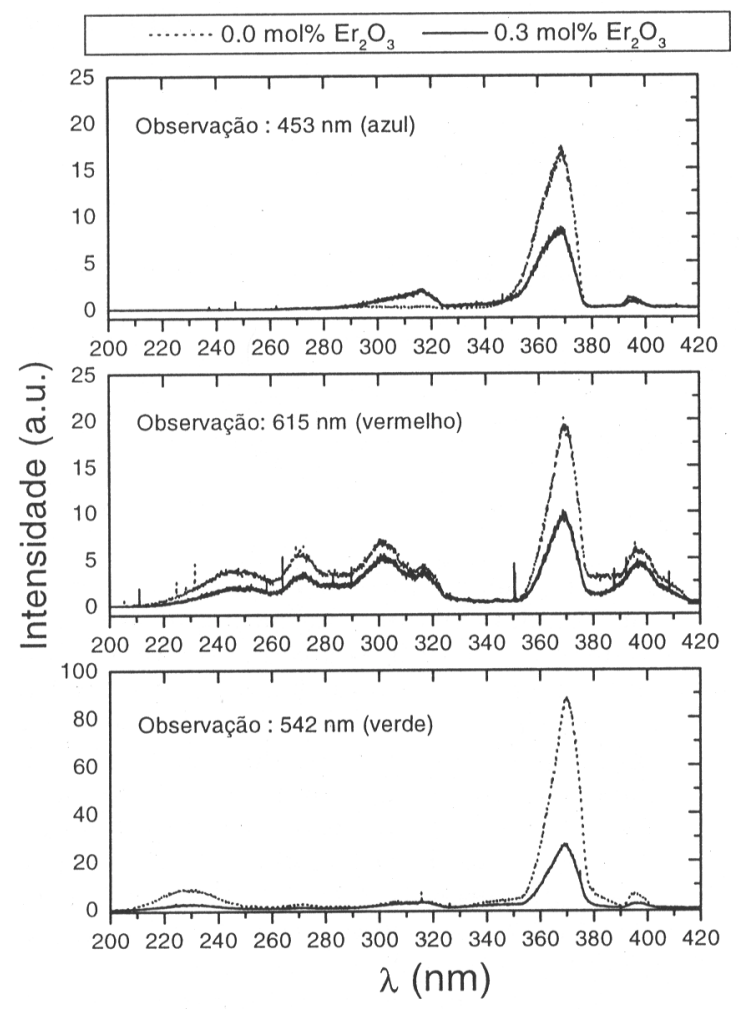

Figura 2. Espectro de excitação do vidro multidopado com 0,3 mol\% de érbio (linha sólida) e sem érbio (linha pontilhada) para observação em $453 \mathrm{~nm}$ - transiçãoo ${ }^{1} \mathrm{D}_{2} \rightarrow{ }^{3} F_{4}(453 \mathrm{~nm})$ do ion $\mathrm{Tm}^{+3}$ (acima), $615 \mathrm{~nm}$ - transição ${ }^{5} \mathrm{D}_{0} \rightarrow{ }^{7} \mathrm{~F}_{2}$ do íon $\mathrm{Eu}^{+3}$ (meio), e em $586 \mathrm{~nm}$ - transição ${ }^{5} \mathrm{D}_{4} \rightarrow \mathrm{F}_{5} \mathrm{~F}_{5}$ do ion $\mathrm{Tb}^{+3}$ (abaixo).

\section{REFERÊNCIAS}

1. Goh, S. C.; J. Non-Cryst. Solids 1993, 161, 227.

2. Dejneka, M.; E. R. E. Riman, J. Lumin. 1995, 65, 227.

3. Silva, J. E. C.; Malta, O. L.; de Sá, G. F.; Santa-Cruz, P. A.; J. Lumin. 1997, 72, 270 e Proc. Int. Conf. on Luminescence - ICL'96, Praga,18 -23 de agosto de 1996. 4. Patente Pendente.

5. Malta, O. L.; Santa-Cruz, P. A.; de Sá, G. F.; Auzel, F.; J. Lumin. 1985, 33, 261. 